International Journal of Advanced Biological and Biomedical Research Available online at http:www.ijabbr.com

Volume 7, Issue 2 (2019) pp. 123-128

Original Article

\title{
Compare Modeling the effect of Flow Parameters on the Efficiency of Membrane Clarification of Pomegranate Juice Regression Method with Artificial Intelligence Methods
}

\author{
Marzieh Toupal Poudineh
}

Department of Food Sciences \& Technology, Faculty of Advanced Sciences \& Technology Pharmaceutical Sciences Branch, Islamic Azad University, Tehran- Iran (IAUPS)

*Corresponding Author E-mail: tp.poudineh@yahoo.com

Received: 10 December 2018, Revised: 15 January 2019, Accepted: 22 January 2019

\begin{abstract}
Objective: Pomegranate juice is a fruit native to Iran because of attractive color, smell and value of mineral water is popular fruits in the world. Pomegranate juice has more nutritional value is due to a combination of anthocyanins that reduce the risk of diseases such as cancer. Processes Feta membrane such as microfiltration 1 and 2 are used to clarify beer industry. Methods: The advantage of this method compared to traditional methods require less labor, higher yields and the process is low. Making a mathematical model or artificial intelligence to predict the juice clarification process in membrane systems is a valuable tool in the field of membrane science and technology. Results: These models play an important role in the simulation and optimization of transparency in membrane systems in order to achieve an economic and efficient design Play. Many of the older models Polar models, the osmotic pressure and boundary layer model have been used to simulate the performance tuning 1 fruit juices. Conclusion: In this study, we tried to take advantage of four regression system, fuzzy inference, neural networks, and fuzzy-neural adaptive method for predicting the flow of water permeate the membrane pomegranate transparency in the system assessed.
\end{abstract}

Key words: Pomegranate juice Processes membrane Mathematical models.

\section{Introduction}

Pomegranate family Pvnyka Tuesday (Punicase granatum L), which is a small tree with prickly branches and leaves are green (Potter et al., 2012). Sphere-shaped fruit with a thick skin and red colors. Pomegranate in Mesopotamia, North Africa, Southern Europe and Spain also been published (Saxena et al., 1987). The data that is presented according to different sources, average fruit weight 150-300 grams, which is about 5066 weight percent aggregate it up. $45-6 \%$ of the total amount of fruit extract is the extract contains $16-17.1 \%$ dry matter is that its ingredients include lipids, organic acids, tannins, various vitamins, minerals soluble, phenolic compounds and enzymes and plant pigments (Withy et al., 1993). In terms of quantity, about $75-80 \%$ by weight 
of water to form fruit. Carbohydrate and crude fiber pomegranate are the most important compounds. The cellulosic material in the core and pomegranate skin than other parts, but the juice is removed cellulosic materials and crude fiber and carbohydrates in beer stays only. Anthocyanins, tannins, organic acids and vitamin $\mathrm{C}$, contain traces of pectin, protein and minerals are the most important constituents of pomegranate juice on taste and color of pomegranate effect. Ingredients of the fruit in different varieties that are as (Table 1), is average (Fadavi et al., 2005). To reduce the volume and reduce the risk of deterioration, fruit juice has been extracted from fruit concentrate and GME is concentrated, thus saving on transport costs and increase shelf life. In this study, we tried to take advantage of four regression system, fuzzy inference, neural networks, and fuzzy-neural adaptive to predict the flow of water permeate the membrane Pomegranate transparency in the system assessed. In this study, we evaluated based on five inputs permeate the membrane, the pore size of the membrane, process pressure, velocity and time based process. Then, according to laboratory-test, regression modeling, using fuzzy inference systems, artificial neural networks, and fuzzy-neural adaptive method will be performed and the function of each of these models to predict the permeate flow parameters evaluation mean square error (MSE), the sum of squared errors (SSE), and the goodness of fit (R2) will be reported. Finally, the best model as an alternative method to estimate the values of input variables related to membrane clearing system without the need for laboratory data will be introduced. Since it is possible to measure all input variables ago-Nose amount of flux is not possible. Sensitivity analysis techniques using artificial neural network method is chosen and flux according to the values of these parameters will be determined.

\section{Materials and Methods}

\section{Permeate flux measurements}

Pomegranate juice consumption Pomegranate Malas, Saveh research was purchased from garden and then peeled using a manual press juice through sieve with a mesh No. 9 was passed. And polyethylene terephthalate one-liter packages for quick-frozen at $-25^{\circ} \mathrm{C}$ until test time was kept.

\section{Membrane processes used}

As seen in the Figure, the first solution in the feed tank was jacketed and was drawn by the pump and the membrane flow. On the track, a barometer to show how much pressure was applied. After passing through the membrane into the permeate solution (that passes through the membrane) and the retentate (not crossed the membrane) was divided. Retentate was also a flow meter on the track and in the end, permeate poured into a container which was placed on the scales and weigh its contents at any moment changes were recorded. (Figure 1), Page module used in this study shows.

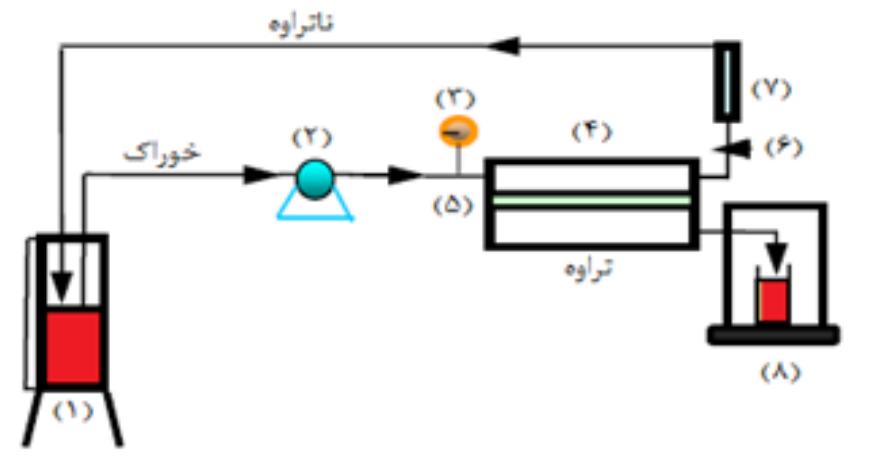

Figure 1. Schematic page module used in this study (1: feed tanks; 2: pump; 3: Barometers; 4: Module plate, 5: membrane; 6: valve; 7: flow meter; 8: container permeate collection) 


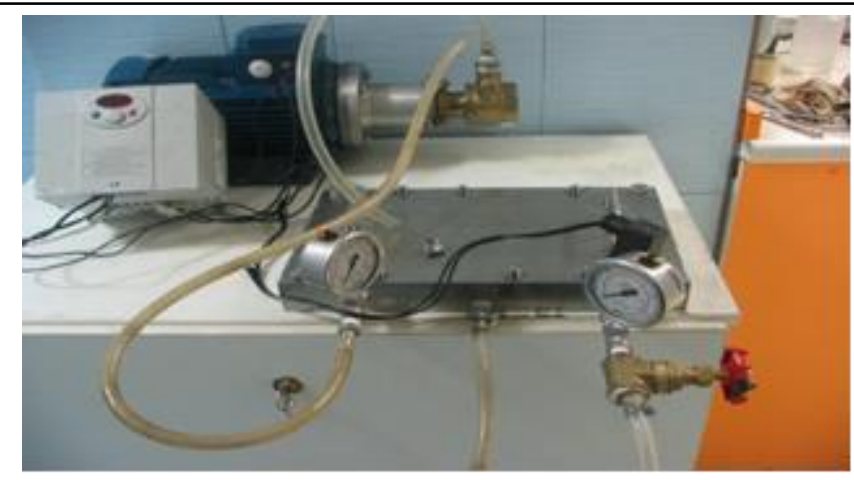

Figure 2. Devices membrane module picture page

Two types of membranes used in the process that the company Millipore membranes (Millipore, United states) was purchased: a) Polyvinylidene fluoride (PVDF) flat membrane with micrometer pore size of 22/0, b). Mixed cellulose ester (MCE) flat membranes with pore size of 025/0,1/0 and 22/0 micrometers.

\section{Regression model (statistical)}

1. A statistical regression analysis to estimate the relationship between variables is the process. It involves many techniques for modeling and analysis of certain variables and is unique, especially when focusing on the relationships between the dependent variable and one or more independent variables. Regression analysis is widely used for forecasting and estimating the dependent variables. In certain circumstances this analysis to infer causal relationships between independent and dependent variables were used (Schoenfeld et al., 1982).

Description: regression equation may be used to describe a certain process or as a model to be used to describe a system of interaction. In this case, try to be the minimum number of independent variables are chosen such that much of the variation in the dependent variable describe.

2. estimates and forecasts: Sometimes regression equation to estimate and predict caused. In this case, using the regression equation the dependent variable for other observations predicted or estimated average of the dependent variable corresponding to a different view. The so-selected variables that predict mean square error is minimized.

3. Control: a regression equation may be used as a means of control. When examining a regression model, the relationship between the independent variable and the dependent variable is predetermined, after determining the appropriate regression equation, coefficient has been tested and validated remains to be assessed. But in many cases, a series of variables that must be included in the regression model, not preset. Studies have shown that independent variables type of membrane, membrane pore size, flow rate, pressure and time the greatest impact on the resulting permeate flux of membrane processes are transparent, As a result, after measuring the amount of pomegranate juice permeate flux conditions in the laboratory, regression model are as follows:

$$
J_{p}=f(M, d, P, V, t)
$$


Where, Jp flux, M type membrane, $\mathrm{d}$ membrane pore size, $\mathrm{P}$ process pressure, $\mathrm{V}$ the speed of the flow, and $t$ is time-process. In this study, we tried optimal model based on equations such as equations, polynomials, power, and exponential data obtained in the laboratory recognized and reported. Regression to model the data in the study of curve fitting software tool was used MATLAB R2015a.

\section{Regression modeling results}

Results of regression modeling based on data from laboratory method of measuring the flux for different values of the independent variables in order to PVDF membranes and MCE are shown in Figure 1-3 and 3-2. In the diagrams of processes that push them more, it seems more and in this case the flux output over time by clogging pores, reduced flux. It was observed that the permeate flux with increasing speed increases. The effect on the flux membranes with pore size and type can be achieved interesting results. But in the final minute process can be seen that the amount of flux for membranes with pore size is smaller. It seems that with time, hard compounds on the surface of the cake layer deposition and the thickness of the membrane increases. MCE hydrophilic membrane leads to clogging of the membrane is in the final minutes and it reduces the amount of permeate flux crossing it.
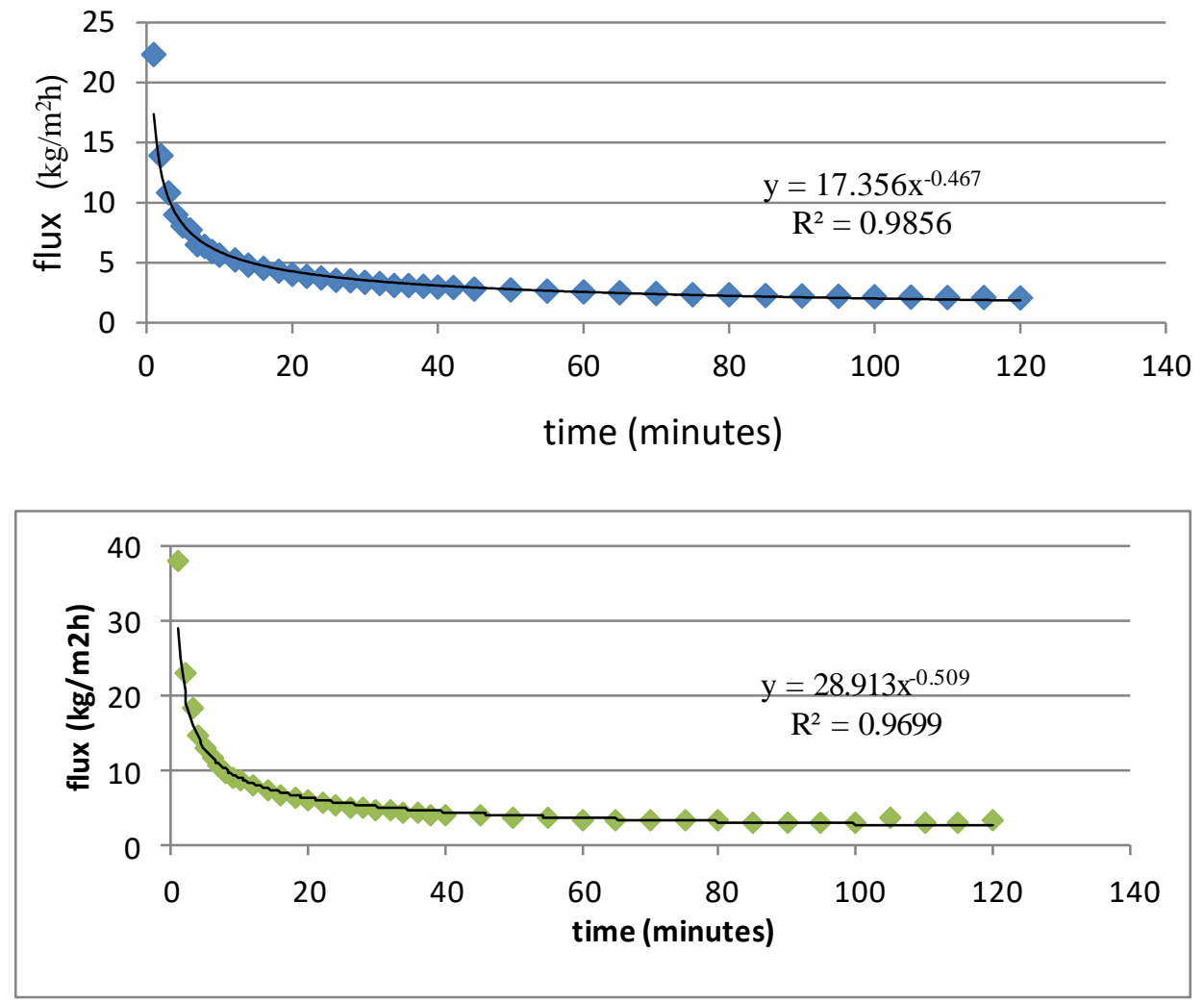

Figure 3. To help flux modeling regression model for different modes for PVDF membranes for (a) speed m/s 095/0, 22/0 pressure of 5.0 bar and a pore size of micrometers (b) speed m/s 095/0 5 bar and micrometer pore size 22/0 (c) speed m/s 533/0, 22/0 micrometer pore size 5 times and pressure 
As is shown on Figure 3, the power regression model was used to fit the data. Good value fit (R2) that are fitted under each of these has been shown, in all cases is more than 95/0 regression model confirmed the results. Thus it can be concluded that for specifying the type of membrane, pore size, flow rate and pressure, the amount of flux since the process obeys a power relationship:

$$
\text { (3-1) } J_{p}=\alpha t^{-\beta}
$$

Statistical parameters and criteria to assess the ability to fit into any of the charts (Figure 1) shows in percentage terms. So, you can regression model with the values of input parameters including type of membrane, pore size, flow rate, and pressure process and the parameters of the equation for power ( $\alpha$ and $\beta$ ) flux predicted with reasonable accuracy. MSE performance parameter values show that in some areas the amount of errors is over the limit. For example, as is clear in the form of flux values at the start of the curves are fitted with a negligible error.

Table 1. statistical parameters and evaluation criteria can fit model $J_{p}=\alpha t^{-\beta}$ (in percent)

\begin{tabular}{cccccccccc}
\hline MAE & SSE & MSE & $\mathbf{R}^{2}$ & $\boldsymbol{\beta}$ & $\boldsymbol{\alpha}$ & Pressure & $\begin{array}{c}\text { Flow } \\
\text { rate }\end{array}$ & $\begin{array}{c}\text { Pore } \\
\text { size }\end{array}$ & $\begin{array}{c}\text { Type } \\
\text { membrane }\end{array}$ \\
\hline 1.15 & 89.5 & 2.13 & $99 \cdot /$ & $47 \cdot /$ & 17.36 & $5 \cdot /$ & $095 \cdot /$ &.$/ 22$ & \\
$97 \cdot /$ & 58.6 & 1.43 & $97 \cdot /$ & $51 \cdot /$ & 28.91 & 5 & $095 \cdot /$ & $22 \cdot /$ & PVDF \\
2.21 & 128 & 3.12 & $98 \cdot /$ & $43 \cdot /$ & 37.31 & 5 & $533 \cdot /$ & $22 \cdot /$ & \\
2.66 & 134.5 & 3.28 & $99 \cdot /$ & $45 \cdot /$ & 14.03 & $5 \cdot /$ & $095 \cdot /$ & $1 \cdot /$ & \\
1.54 & 117.3 & 2.86 & $99 \cdot /$ & $46 \cdot /$ & 19.66 & 2 & $095 \cdot /$ & $1 \cdot /$ & \\
$76 \cdot /$ & 81.06 & 1.99 & $98 \cdot /$ & $44 \cdot /$ & 20.05 & 5 & $095 \cdot /$ & $1 \cdot /$ & \\
1.94 & 91 & 2.22 & $98 \cdot /$ & $46 \cdot /$ & 41.51 & 5 & $533 \cdot /$ & $1 \cdot /$ & MCE \\
$77 \cdot /$ & 52.9 & 1.29 & $97 \cdot /$ & $31 \cdot /$ & 12.41 & $5 \cdot /$ & $095 \cdot /$ & $025 \cdot /$ & \\
1.55 & 97.6 & 2.38 & $99 \cdot /$ & $62 \cdot /$ & 32.09 & $5 \cdot /$ & $095 \cdot /$ & $22 \cdot /$ & \\
\hline
\end{tabular}

using four regression modeling approach, using fuzzy inference system, artificial neural networks, adaptive neuro-fuzzy methods were used. As can be seen, regression model despite the simplicity of algorithms, data modeling was a good performance, but it should be borne in mind that the nature of this method is such that all data in the system learning, ittaught.

Check (Table 2) shows the overall system error in predicting flux for membrane pre-MCE less than forecast error in flux for the PVDF membrane. This may be because more of the kind of analysis that compared MCE membrane with the membrane PVDF, artificial intelligence techniques to extract patterns in the data are better.

Table 2. Performance Forecast for systems and optimal experimental data (in percent)

\begin{tabular}{ccccccc}
\hline & MCE & & \multicolumn{3}{c}{ PVDF } & \multirow{2}{*}{ Name System } \\
MAE & SSE & MSE & MAE & SSE & MSE & Regression \\
\hline $0 / 77$ & $52 / 9$ & $1 / 29$ & $0 / 97$ & $58 / 6$ & $1 / 43$ & R
\end{tabular}


Adaptive neuro-fuzzy system designed in this study can be used in the manufacture of fruitjuice used in industry and research centers. The benefits of neuro-fuzzy modeling performed in this study can be understood and noted for its simplicity of implementation. To provide a model of its efficient use and derive tangible output. The results of this study with previous research was similar. The results of this study with the results Noorbakhsh and colleagues (Nourbakhsh et al., 2014), a three-layered artificial neural network model to predict the discharge of juice in the process transparent membrane designed the match. Membrane transport model input parameters including pressure, temperature, speed, pore size and time. They showed the best neural network architecture to predict Dubai juice under review, the structure was in this study, similar results were obtained for pomegranate juice (Nourbakhsh et al., 2014). Also, according to the results obtained in this study, has been shown in several studies in the field of food industry that the performance of systems Neuro-fuzzy artificial neural network approach has better performance compared to (Taghadomi et al., 2014).

\section{References}

Fadavi, A, Barzegar, M, Azizi, MH, Bayat, M, (2005). Note. Physicochemical composition of ten pomegranate cultivars (Punica granatum L.) grown in Iran. Food Sci Technol Int, Apr 1; 11:113-9.

Nourbakhsh, H, Emam-Djomeh, Z, Omid, M, Mirsaeedghazi, H, Moini, S, (2014). Prediction of red plum juice permeate flux during membrane processing with ANN optimized using RSM. Comput Electron Agric, 31:102:1-9.

Potter, NN, Hotchkiss, JH, (2012). Food science. Springer Science \& Business Media, Dec 6.

Saxena, AK, Manan, JK, Berry, SK, (1987). Pomegranate: Post harvest technology, chemistry and processing. Indian Food Packer, 41:43.

Schoenfeld, D, (1982). Partial residuals for the proportional hazards regression model. Biometrika, 69:239-41.

Taghadomi-Saberi, S, Omid, M, Emam-Djomeh, Z, Ahmadi, H, (2014). Evaluating the potential of artificial neural network and neuro-fuzzy techniques for estimating antioxidant activity and anthocyanin content of sweet cherry during ripening by using image processing. J Sci Food Agric, 94:95-101.

Withy, LM, Nguyen, TT, Wrolstad, RE, Heatherbell, DA, (1993). Storage changes in anthocyanin content of red raspberry juice concentrate. J food Sci, 58:190-2.

How to cite this article: Marzieh Toupal Poudineh, Compare Modeling the effect of Flow Parameters on the Efficiency of Membrane Clarification of Pomegranate Juice Regression Method with Artificial Intelligence Methods. International Journal of Advanced Biological and Biomedical Research, 2019, 7(2), 123-128. http://www.ijabbr.com/article 34098.html 